\title{
Uncovering the Mechanism of Astragalus membranaceus in the Treatment of Diabetic Nephropathy Based on Network Pharmacology
}

\author{
Ming-Fei Guo $\mathbb{D}^{1},{ }^{1}$ Ya-Ji Dai $\mathbb{D}^{2},{ }^{2}$ Jia-Rong Gao, ${ }^{3}$ and Pei-Jie Chen ${ }^{1}$ \\ ${ }^{1}$ Department of Pharmacy, The Fourth Affiliated Hospital of Anhui Medical University, Hefei, Anhui 230012, China \\ ${ }^{2}$ Department of Pharmacy, Anhui No.2 Provincial People's Hospital, Hefei, Anhui 230041, China \\ ${ }^{3}$ Department of Pharmacy, The First Affiliated Hospital of Anhui University of Chinese Medicine, Hefei, Anhui 230031, China
}

Correspondence should be addressed to Ya-Ji Dai; dyj920914@163.com

Received 8 September 2019; Revised 15 January 2020; Accepted 6 February 2020; Published 6 March 2020

Academic Editor: Ilaria Campesi

Copyright (c) 2020 Ming-Fei Guo et al. This is an open access article distributed under the Creative Commons Attribution License, which permits unrestricted use, distribution, and reproduction in any medium, provided the original work is properly cited.

\begin{abstract}
Background. Diabetic nephropathy (DN), characterized by hyperglycemia, hypertension, proteinuria, and edema, is a unique microvascular complication of diabetes. Traditional Chinese medicine (TCM) Astragalus membranaceus (AM) has been widely used for DN in China while the pharmacological mechanisms are still unclear. This work is aimed at undertaking a network pharmacology analysis to reveal the mechanism of the effects of AM in DN. Materials and Methods. In this study, chemical constituents of AM were obtained via Traditional Chinese Medicine Systems Pharmacology Database (TCMSP), and the potential targets of AM were identified using the Therapeutic Target Database (TTD). DisGeNET and GeneCards databases were used to collect DN-related target genes. DN-AM common target protein interaction network was established by using the STRING database. Gene Ontology (GO) and Kyoto Encyclopedia of Genes and Genomes (KEGG) pathway enrichment analyses were carried out to further explore the DN mechanism and therapeutic effect of AM. The network diagrams of the active component-action target and protein-protein interaction (PPI) networks were constructed using Cytoscape software. Results. A total of 16 active ingredients contained and 78 putative identified target genes were screened from AM, of which 42 overlapped with the targets of DN and were considered potential therapeutic targets. The analysis of the network results showed that the AM activity of component quercetin, formononetin, calycosin, 7-O-methylisomucronulatol, and quercetin have a good binding activity with top ten screened targets, such as VEGFA, TNF, IL-6, MAPK, CCL3, NOS3, PTGS2, IL-1 $\beta$, JUN, and EGFR. GO and KEGG analyses revealed that these targets were associated with inflammatory response, angiogenesis, oxidative stress reaction, rheumatoid arthritis, and other biological process. Conclusions. This study demonstrated the multicomponent, multitarget, and multichannel characteristics of AM, which provided a novel approach for further research of the mechanism of $\mathrm{AM}$ in the treatment of $\mathrm{DN}$.
\end{abstract}

\section{Introduction}

The etiology and pathogenesis of diabetic nephropathy (DN) are complex and diverse [1]. There are many factors involved, such as infection, autoimmunity, genetics, drugs, diet, and environment $[2,3]$. The specific pathogenesis is still not fully clear. The incidence of DN accounts for $20 \%-40 \%$ of all diabetic patients, and it is also one of the main causes of death [4]. DN is characterized by edema, hyperglycemia, hypertension, and albuminuria. With the deterioration of
$\mathrm{DN}$, it will eventually lead to glomerulosclerosis and renal failure. Therefore, lowering the blood sugar, decreasing blood pressure, and alleviation of renal microvascular damage are crucial for the treatment of DN.

Traditional Chinese medicine (TCM) has been playing an important role in the improvement of life quality and curing disease. In recent years, much attention has been focused on the Chinese herb medicine for the treatment of diabetes and metabolic syndrome. Astragalus membranaceus (AM) has a longstanding history and has gained 


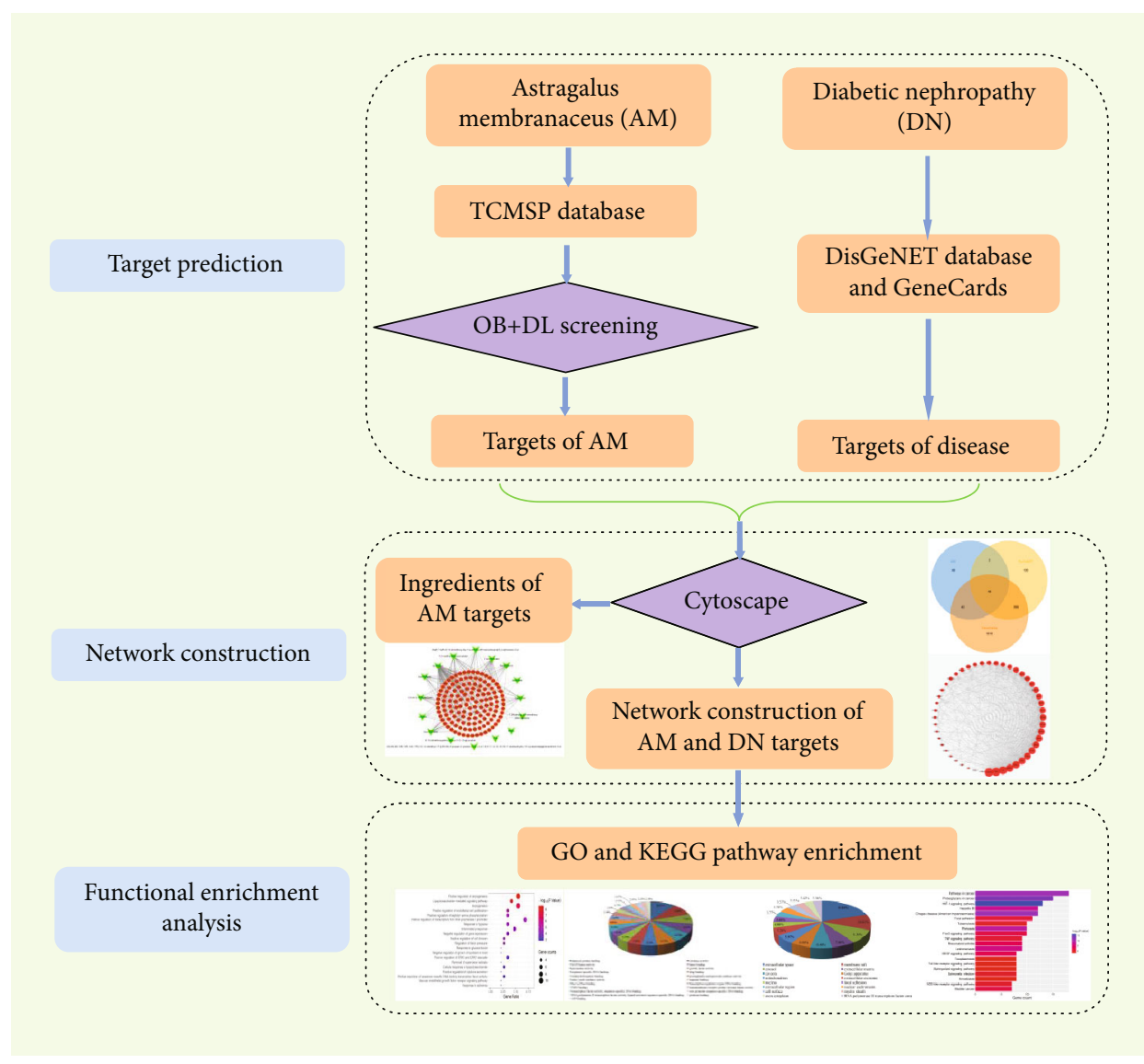

FIGURE 1: The whole framework based on an integration strategy of network pharmacology.

widespread clinical applications in China, with the functions of lowering blood sugar, decreasing blood lipid, eliminating edema, antioxidative stress, and so on $[5,6]$. In addition, it has been proven to have wide pharmacological effects on diabetic diseases; particularly in DN aspects, it has a unique curative effect $[7,8]$.

It is well known that TCM is guided by the theory of TCM and characteristics of being multicomponent, multitarget, and multipathway in the treatment of diseases. AM therapeutic effects on DN by regulating Nrf2/HO-1 signaling pathway, antioxidant stress, nuclear factor-kappa B, and immunoregulation were reported [9, 10]. However, the pharmacological mechanisms and material bases of the action of AM related to DN remain elusive. Accordingly, an increasing number of researchers are keen to explore comprehensive and systematic evaluation of the pharmacological mechanism of AM on DN which is urgently needed.

Network pharmacology is an interactive network based on the drug-target-gene-disease, including chemoinformatics, bioinformatics, network biology, and pharmacology $[11,12]$. It has become a comprehensive tool to systematically reveal the complex network relationships between the bioactive components and potential mechanisms of TCM formulas from a systemic perspective [13]. The purpose of this study was to explore the potential mechanism of AM on DN disease by using network pharmacology, drug- targeted interaction databases, and biological analysis methods. Our flowchart is shown in Figure 1.

\section{Materials and Methods}

2.1. Database Building and Active Compound Screening. Traditional Chinese Medicine System Pharmacology Database (TCMSP, http://lsp.nwu.edu.cn/tcmsp.php) was used to collect the chemical constituents of AM; it was a unique system pharmacology platform that captures the relationships between herbal ingredients, targets, and diseases $[14,15]$. It is constructed based on scientific publications and medical literature on TCM, which contains more than 13,731 pure compounds isolated from 505 TCM herbs. Twelve pharmacokinetic parameters can be provided, such as oral bioavailability (OB), half-life (HL), drug-likeness (DL), Caco-2 permeability (Caco-2), and blood-brain barrier (BBB). We screened AM compounds based on absorption, distribution, metabolism, and excretion (ADME), and pharmacokinetic information retrieval filters were used to retrieve bioactive compounds for further analysis under the conditions of $\mathrm{OB} \geq 30 \%$ and $\mathrm{DL} \geq 0.18[16,17]$.

2.2. Screening of Potential Targets for DN. Information on $\mathrm{DN}$-associated target genes were gathered from the following database. DisGeNET (https://www.disgenet.org/home/), a comprehensive multifunctional data platform that contains 
TABLE 1: Basic information of AM compound ingredients.

\begin{tabular}{|c|c|c|c|}
\hline Mol ID & Molecule name & $\mathrm{OB}(\%)$ & $\mathrm{DL}$ \\
\hline MOL000211 & Mairin & 55.38 & 0.78 \\
\hline MOL000239 & Jaranol & 50.83 & 0.29 \\
\hline MOL000296 & Hederagenin & 36.91 & 0.75 \\
\hline MOL000033 & $\begin{array}{l}\text { (3S,8S,9S,10R,13R,14S,17R)-10,13-Dimethyl-17- } \\
{[(2 \mathrm{R}, 5 \mathrm{~S}) \text {-5-propan-2-yloctan-2-yl]-2,3,4,7,8,9,11,12,14,15,16,17- }} \\
\text { dodecahydro-1H-cyclopenta[a]phenanthren-3-ol }\end{array}$ & 36.23 & 0.78 \\
\hline MOL000354 & Isorhamnetin & 49.6 & 0.31 \\
\hline MOL000371 & 3,9-Di-O-methylnissolin & 53.74 & 0.48 \\
\hline MOL000379 & 9,10-Dimethoxypterocarpan-3-O- $\beta$-D-glucoside & 36.74 & 0.92 \\
\hline MOL000380 & $\begin{array}{l}\text { (6aR,11aR)-9,10-Dimethoxy-6a,11a-dihydro-6H- } \\
\text { benzofurano[3,2-c] chromen-3-ol }\end{array}$ & 64.26 & 0.42 \\
\hline MOL000387 & Bifendate & 31.1 & 0.67 \\
\hline MOL000392 & Formononetin & 69.67 & 0.21 \\
\hline MOL000398 & 7-O-methylisomucronulatol & 74.69 & 0.3 \\
\hline MOL000417 & Calycosin & 47.75 & 0.24 \\
\hline MOL000422 & Kaempferol & 41.88 & 0.24 \\
\hline MOL000433 & FA & 68.96 & 0.71 \\
\hline MOL000442 & 1,7-Dihydroxy-3,9-dimethoxy pterocarpene & 39.05 & 0.48 \\
\hline MOL000098 & Quercetin & 46.43 & 0.28 \\
\hline
\end{tabular}

integrated diseases, genes, and experimental research [18]. The GeneCards (https://www.genecards.org/) as a comprehensive database of functions including genomics, proteomics, and transcriptomics [19]. Therefore, we searched in these two databases with the keywords "Diabetic nephropathy" to screen the targets related to DN. The names of targets and their ID were searched on Therapeutic Target Database (TTD) (http://db.idrblab.org/ttd/). It provides information regarding the known and explored therapeutic protein targets, the targeted disease, and the corresponding ID at each of the targets.

2.3. Collecting Compound-Disease Common Targets. The screened chemical targets and disease targets database were imported into the ImageGP (http://www.ehbio.com/ ImageGP/index.php) platform for analysis, and the common targets of compound-disease were obtained as the potential targets for further analysis [20].

2.4. PPI Network Map of Compound-Disease Common Targets. Protein-protein interaction (PPI) network was derived based on STRING database (https://string-db.org/), which covered almost all functional interactions between the expressed proteins [21]. Species are set as "Homo sapiens" and the target interaction information was obtained according to the results of analysis. The results were imported into the Cytoscape (version 3.6.1; https://www.cytoscape.org/) software where the interaction network was drawn and analyzed. The node size was used to reflect the number of combined targets (degree), and the degree greater than twice the median degree of all nodes was selected as a hub according to our previous studies.
2.5. Construction of Active Component-Target Network. A visual network was established through Cytoscape software to reflect the complex relationship between active compounds and potential targets [22]. Nodes represent the compounds and targets, while edges indicate the intermolecular interactions between compounds and targets.

2.6. Gene Ontology (GO) and KEGG Pathway Enrichment Analysis. The biological process (BP), molecular function (MF), cell component (CC), and Kyoto Encyclopedia of Genes and Genomes (KEGG) database (https://www.kegg.jp/) pathway enrichment analysis were using the DAVID system (https://david.ncifcrf.gov/) [23, 24]. In this research, GO functional annotation and KEGG pathway enrichment analyses were performed using $\mathrm{R}$ package, and the $P$ value less than 0.05 was employed for further analysis.

\section{Results}

3.1. Active Compounds of AM. The active compound targets of AM were searched via the TCMSP databases for each chemical component. Eighty-seven compounds were collected from the TCMSP, with the values of $\mathrm{OB} \geq 30 \%$ and $\mathrm{DL} \geq 0.18$ properties, applied to screen the active compounds from AM. At last, 16 candidate ingredients were selected out from AM compounds (Table 1). In total, 142 targets were identified after removing the duplicate data (Supplementary Table S1).

3.2. Compound-Target Network. To further understand the interaction relationship between the compounds and their corresponding targets, we constructed a compound-target network, as shown in Figure 2. By mapping 16 compounds 


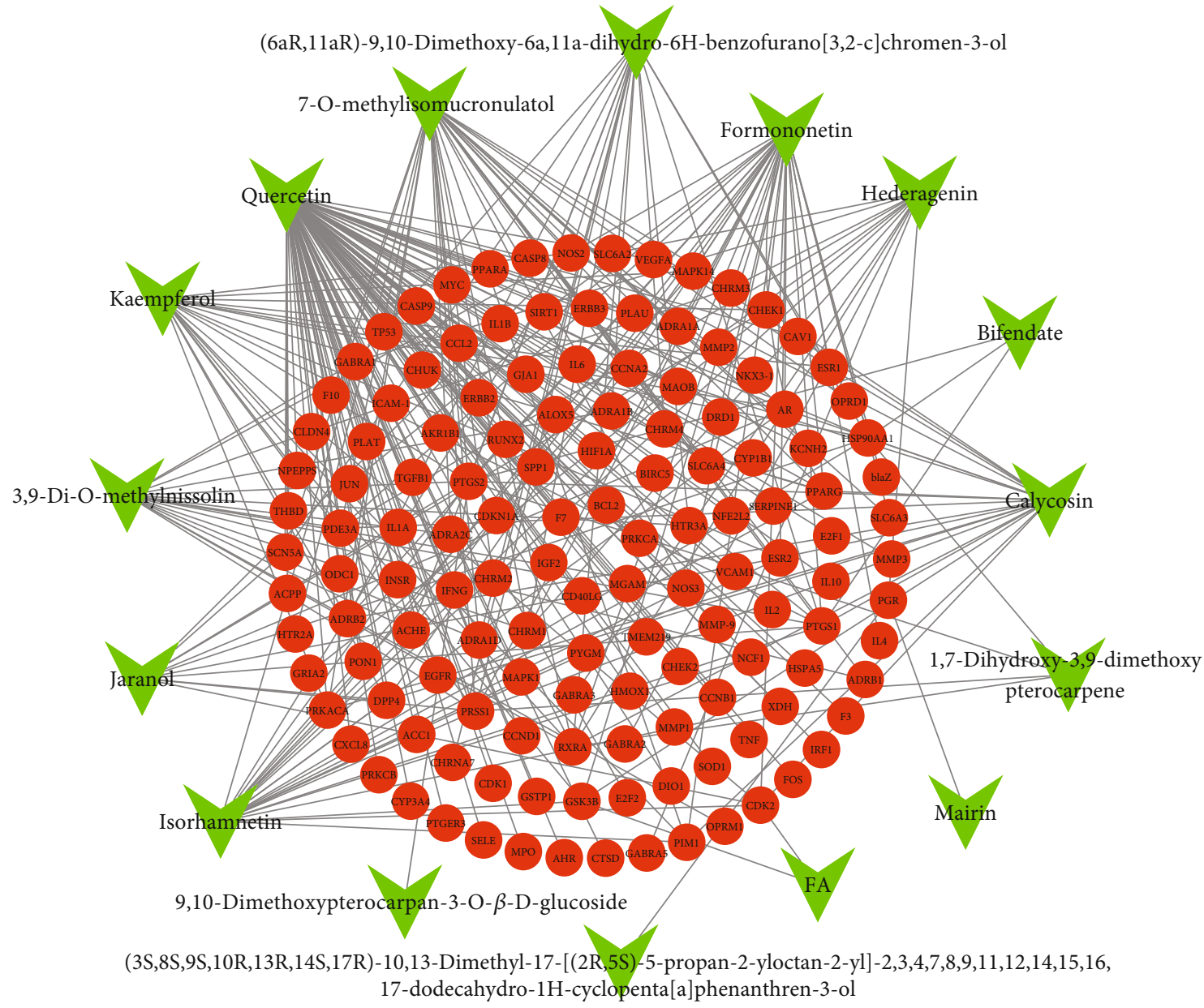

Figure 2: Compound-target network of potential targets in AM. The light green nodes represent the potential active ingredients in AM, and the red nodes represent the corresponding targets of the ingredients.

to 142 potential targets which associated with antioxidant stress, nuclear factor-kappa B, and immune regulation. Based on the docking results, the network embodied 151 nodes and 296 edges, in which the colorized red circles correspond to the targets and the compounds calculated in green from AM. The compounds with more targets are quercetin, 7-Omethylisomucronulatol, formononetin, and isorhamnetin, which correspond to 99, 32, 32, and 23 targets, respectively. The result suggested that these four components probably served as significant therapeutic compounds in DN.

3.3. Retrieval of Potential Disease Targets. Through the DisGeNET and GeneCards databases, the retrieved results were integrated to obtain the DN-related disease protein targets. As shown in Figure 3, the potential target gene in AM was mapped to the disease target gene using the ImageGP platform, and a Venn diagram was drawn. A total of 48 potential targets were obtained based on the intersection of protein targets acting on chemical components and these are related to DN (Table 2). The information regarding these targets is provided in Supplementary Table S2.

3.4. Conversion of Target Proteins into Network and Analysis. A total of 48 potential genes associated with $\mathrm{DN}$ were uploaded to the STRING database for analysis. The systematically selected protein targets with a medium confidence score of 0.400 were plotted as an interaction network. The network of protein-protein interactions (PPI) (Figure 4) was established through the STRING database. From the analysis results, a total of 48 nodes and 539 edges were acquired, and the average node degree is 22.5 . The results were used for further analysis through Cytoscape software, and the network was constructed as Figure 5. The edges represent the association between a pair of action targets, the nodes represent the action target, and the degree value represents its action intensity. The top ten targets VEGFA, TNF, IL-6, MAPK1, CCL2, PTGS2, IL-1 $\beta$, NOS3, JUN, and EGFR have higher degree in this process, which explained their significance in the network.

3.5. Gene Ontology Enrichment Analysis. We imported the selected potential 48 target genes into the DAVID system for GO analysis. The results revealed that the functions of these potential targets are related to many biological processes, molecular function, and cellular components. It may be important for the occurrence and development of DN. A total of 139 biological processes were enrichment, and the top 20 remarkably enriched BP terms were selected for 


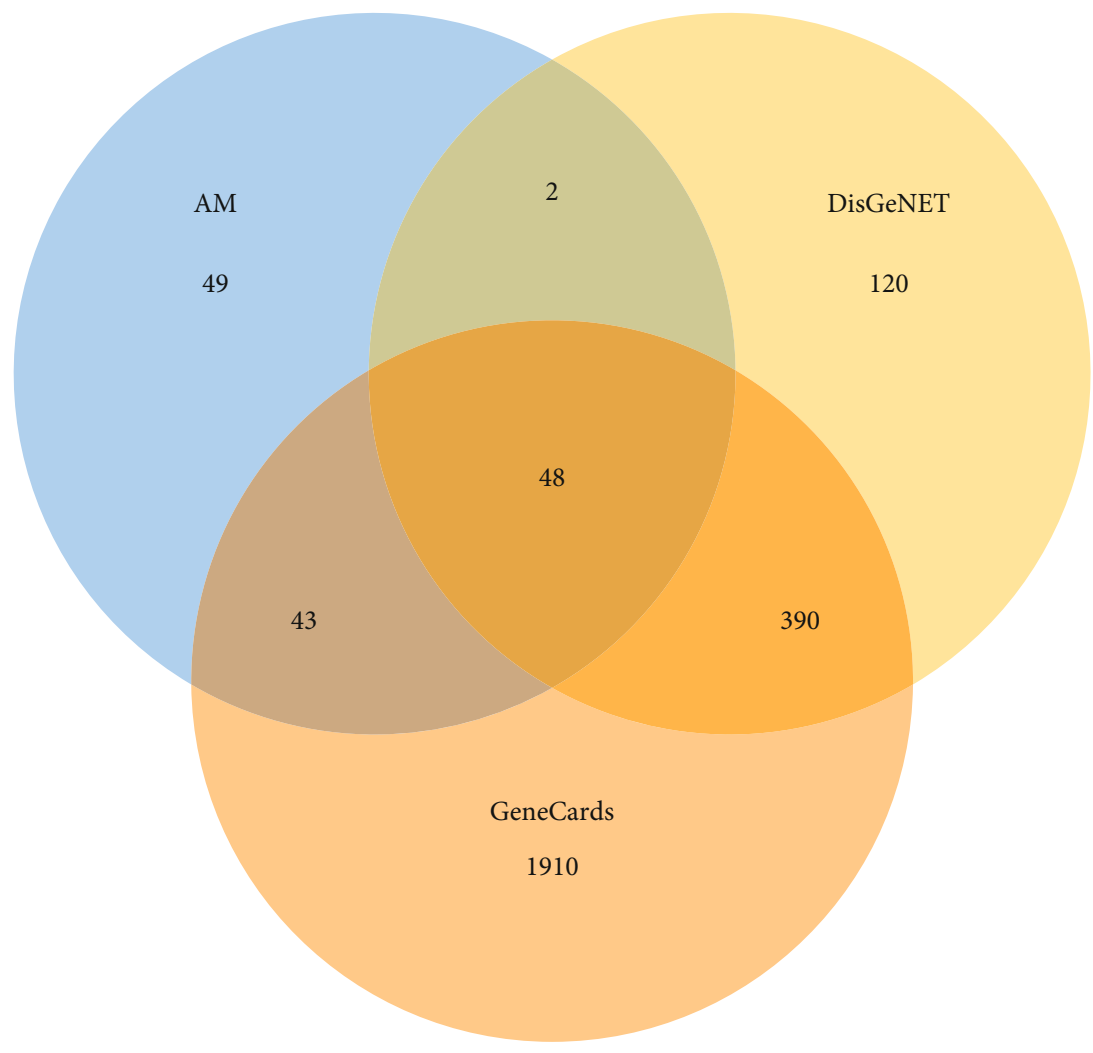

Figure 3: Matching of target genes between DN and AM.

analysis, such as positive regulation of angiogenesis, inflammatory response, and removal of superoxide radicals (Figure 6). The results indicated that AM may play a vital role in the treatment of DN by manipulating these biological processes. These processes are of great significance to further understand the mechanism of AM on the DN.

A total of 21 molecular function (Figure 7) GO terms were enrichment, and the top 13 entries were selected based on $P<0.05$. These targets of molecular function mainly involved identical protein binding, cytokine activity, MAP kinase activity, heme binding, peroxidase activity, growth factor activity, and many genes related to the molecular functions described above.

In all, 16 cell component (Figure 8) GO terms were enrichment, and the top 12 entries were selected based on $P<0.05$. The targets are closely related in extracellular space, membrane raft, cytosol, extracellular matrix, caveola, Golgi apparatus, mitochondrion, and extracellular exosome, and many targets were ranked highly as potential related genes.

3.6. KEGG Pathway Enrichment Analysis. To further reveal the potential mechanism of the AM on the effect of DN, we conducted KEGG pathway enrichment analysis on 48 targets and screened out top 20 pathways based on the threshold of $P<0.01$ (Figure 9). Numerous pathways for potential target genes were identified, such as VEGF signaling pathway, HIF-1 signaling pathway, and FoxO signaling pathways which are associated with signal transduction. Rheumatoid arthritis and TNF signaling pathways are related to the inflammatory reaction process. Bladder cancer and proteoglycans play a crucial role in the pathways in cancer. Moreover, toll-like receptor signaling pathway and focal adhesion are closely related to immunological stress. In addition, we found some other pathways such as leishmaniasis, salmonella infection, amoebiasis, and tuberculosis, which revealed that AM has a potential application in other related diseases. The results certificated that AM alleviated the DN disease by regulating antioxidant stress, immunization, and inflammatory reaction.

\section{Discussion}

Diabetic nephropathy (DN) is one of the common chronic microvascular complications in diabetes mellitus. At present, the incidence of DN is increasing year by year, which seriously threatens the prognosis and quality of life in diabetic patients [25]. The occurrence and development of DN are related to the disorder of glucose and lipid metabolism, oxidative stress, inflammatory reaction, and abnormal vasoactive substances [26, 27]. In this study, the TCM-active ingredient-target network showed that the main active ingredient of AM quercetin, 7-O-methylisomucronulatol, formononetin, and isorhamnetin plays an important role in the network, indicating that they have potential research value in the treatment of DN.

Evidence showed that some compounds found in this study not only have anti-immune and antistress effects but also have the effect of alleviating endocrine and metabolic disorders. It was reported that quercetin could inhibit 
TABLE 2: Information on potential targets and the topological attributes.

\begin{tabular}{|c|c|c|c|c|}
\hline No. & Gene name & Protein name & UniProt ID & Degree \\
\hline 1 & VEGFA & Vascular endothelial growth factor A & P15692 & 41 \\
\hline 2 & TNF & Tumor necrosis factor & P01375 & 40 \\
\hline 3 & IL-6 & Interleukin-6 & P05231 & 39 \\
\hline 4 & MAPK1 & Mitogen-activated protein kinase 1 & P28482 & 37 \\
\hline 5 & CCL2 & C-C motif chemokine 2 & P13500 & 35 \\
\hline 6 & PTGS2 & Alcohol dehydrogenase $1 \mathrm{~B}$ & P35354 & 34 \\
\hline 7 & IL- $1 \beta$ & Interleukin-1 beta & P01584 & 34 \\
\hline 8 & NOS3 & Nitric oxide synthase, endothelial & P29474 & 34 \\
\hline 9 & JUN & Transcription factor AP-1 & P05412 & 34 \\
\hline 10 & EGFR & Epidermal growth factor receptor & P00533 & 33 \\
\hline 11 & MAPK8 & Mitogen-activated protein kinase 8 & P45983 & 33 \\
\hline 12 & IL-10 & Interleukin-10 & P22301 & 31 \\
\hline 13 & PPARG & Peroxisome proliferator-activated receptor gamma & P37231 & 31 \\
\hline 14 & CXCL8 & Interleukin-8 & P10145 & 31 \\
\hline 15 & MMP2 & $72 \mathrm{kDa}$ type IV collagenase & P08253 & 30 \\
\hline 16 & ESR1 & Estrogen receptor & P03372 & 30 \\
\hline 17 & SERPINE1 & Plasminogen activator inhibitor 1 & P05121 & 30 \\
\hline 18 & TGFB1 & Transforming growth factor beta-1 & P01137 & 28 \\
\hline 19 & SIRT1 & NAD-dependent deacetylase sirtuin-1 & Q96EB6 & 28 \\
\hline 20 & MAPK14 & Mitogen-activated protein kinase 14 & Q16539 & 28 \\
\hline 21 & HIF- $1 \alpha$ & Hypoxia-inducible factor 1-alpha & Q16665 & 28 \\
\hline 22 & CAV1 & Caveolin-1 & Q03135 & 26 \\
\hline 23 & SPP1 & Osteopontin & P10451 & 25 \\
\hline 24 & MPO & Myeloperoxidase & P05164 & 25 \\
\hline 25 & MMP3 & Stromelysin-1 & P08254 & 24 \\
\hline 26 & KDR & Vascular endothelial growth factor receptor 2 & P35968 & 24 \\
\hline 27 & NOS2 & Nitric oxide synthase, inducible & P35228 & 23 \\
\hline 28 & SOD1 & Superoxide dismutase $(\mathrm{Cu}-\mathrm{Zn})$ & P00441 & 23 \\
\hline 29 & NFE2L2 & Nuclear factor erythroid 2-related factor 2 & Q16236 & 21 \\
\hline 30 & GJA1 & Gap junction alpha-1 protein & P17302 & 20 \\
\hline 31 & CDKN1A & Cyclin-dependent kinase inhibitor 1 & P38936 & 19 \\
\hline 32 & IL-1A & Interleukin-1 alpha & P01583 & 16 \\
\hline 33 & PRKCA & Protein kinase $\mathrm{C}$ alpha type & P17252 & 15 \\
\hline 34 & PPARA & Peroxisome proliferator-activated receptor alpha & Q07869 & 14 \\
\hline 35 & IGF2 & Insulin-like growth factor II & P01344 & 14 \\
\hline 36 & PTGS1 & Prostaglandin G/H synthase 1 & P23219 & 13 \\
\hline 37 & HSPA5 & $78 \mathrm{kDa}$ glucose-regulated protein & P11021 & 12 \\
\hline 38 & DPP4 & Dipeptidyl peptidase IV & $\mathrm{P} 27487$ & 11 \\
\hline 39 & PRKCB & Protein kinase $C$ beta type & P05771 & 10 \\
\hline 40 & THBD & Thrombomodulin & P07204 & 10 \\
\hline 41 & AKR1B1 & Aldose reductase & P15121 & 8 \\
\hline 42 & PON1 & Serum paraoxonase/arylesterase 1 & P27169 & 8 \\
\hline 43 & INSR & Insulin receptor & P06213 & 8 \\
\hline 44 & BCL2 & Apoptosis regulator $\mathrm{Bcl}-2$ & P10415 & 7 \\
\hline 45 & $\mathrm{E} 2 \mathrm{~F} 1$ & Transcription factor E2F1 & Q01094 & 6 \\
\hline 46 & LYZ & Lysozyme & P61626 & 5 \\
\hline 47 & HTR2A & 5-hydroxytryptamine $2 \mathrm{~A}$ receptor & P28223 & 1 \\
\hline 48 & PRSS1 & Trypsin-1 & P07477 & 1 \\
\hline
\end{tabular}




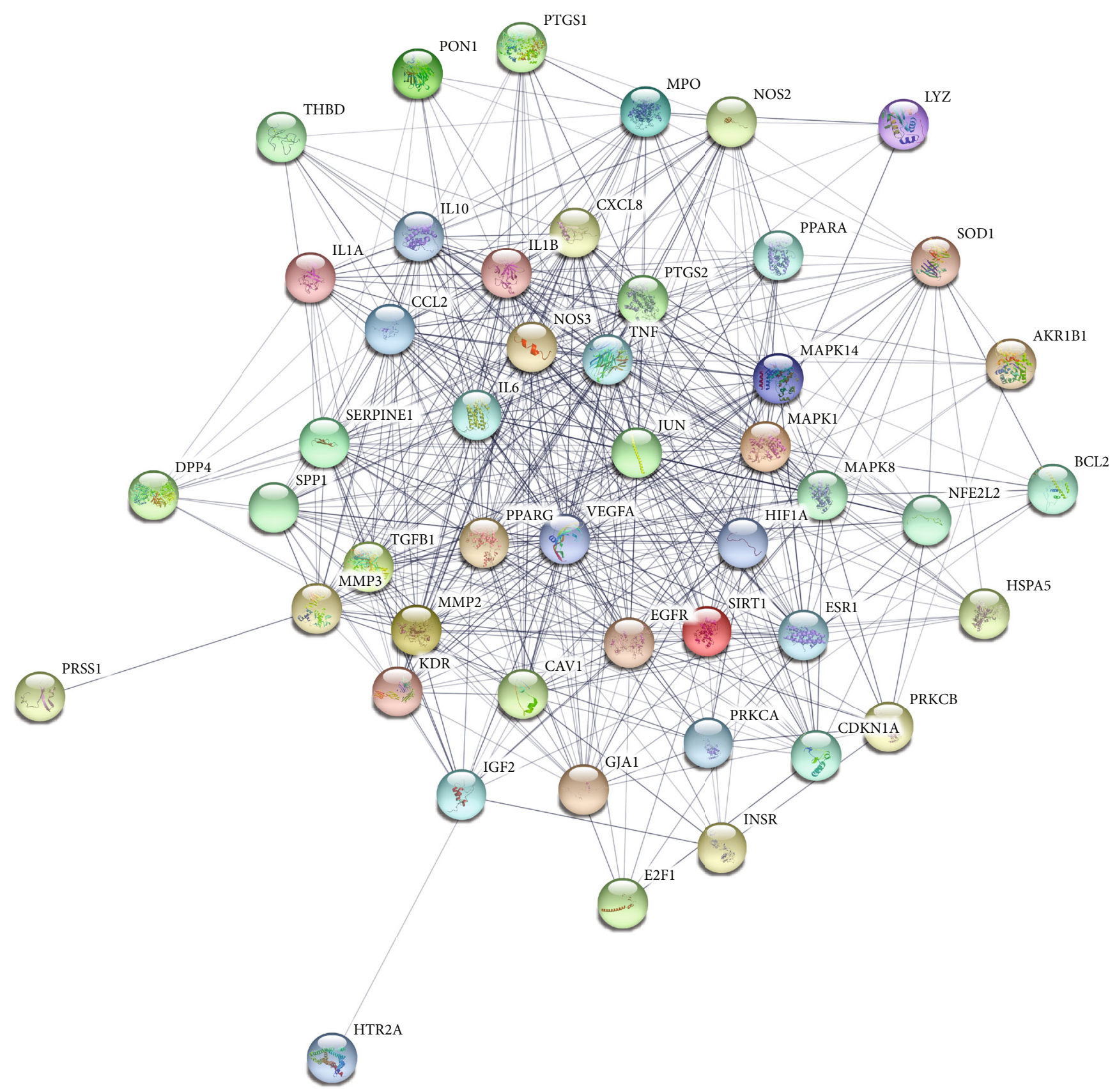

Figure 4: Common target PPI network between DN and AM. Each bubble node represents a protein, and the 3D structure in the bubble nodes represent that the protein spatial structure is known or predicted. The lines among inner nodes display the relationship between different proteins, and the width of lines was based on the strength of data support.

glomerular mesangial cell proliferation in high glucosetreated DN mouse and via reactivation of the Hippo pathway [28]. Meanwhile, quercetin can increase the SIRT1 expression and resistance to oxidative stress [29]. Previous studies have suggested that formononetin could significantly decrease the HIF-1 and VEGF protein expression level, which is widely used in the field of cardiovascular diseases [30, 31].

As we can see from the compound-target network, many targets can be adjusted by multiple compounds, such as NOS3, PTGS2, JUN, MAPK1, and CCL2. Meanwhile, MAPK1, SOD1, TGFB1, and others can be only regulated by quercetin. It indicated that AM has the biological charac- teristics of multicomponent and multitarget in treating DN. Common target PPI network showed that the targets are also controlled by AM and DN, which revealed that AM could regulate the expression of DN-regulated targets and alleviate DN symptoms. VEGFA (degree $=41)$, TNF $($ degree $=40)$, IL-6 $\quad($ degree $=39)$, MAPK1 $\quad($ degree $=37)$ and CCL2 $($ degree $=35)$ might be the hub target of this network.

In order to predict the mechanism of AM in the treatment of DN, we analyzed the candidate targets by performing GO enrichment results, such as biological processes, molecular function, and cellular components. The top 20 GO terms $(P<0.05)$ were chosen from the 138 enrichment 


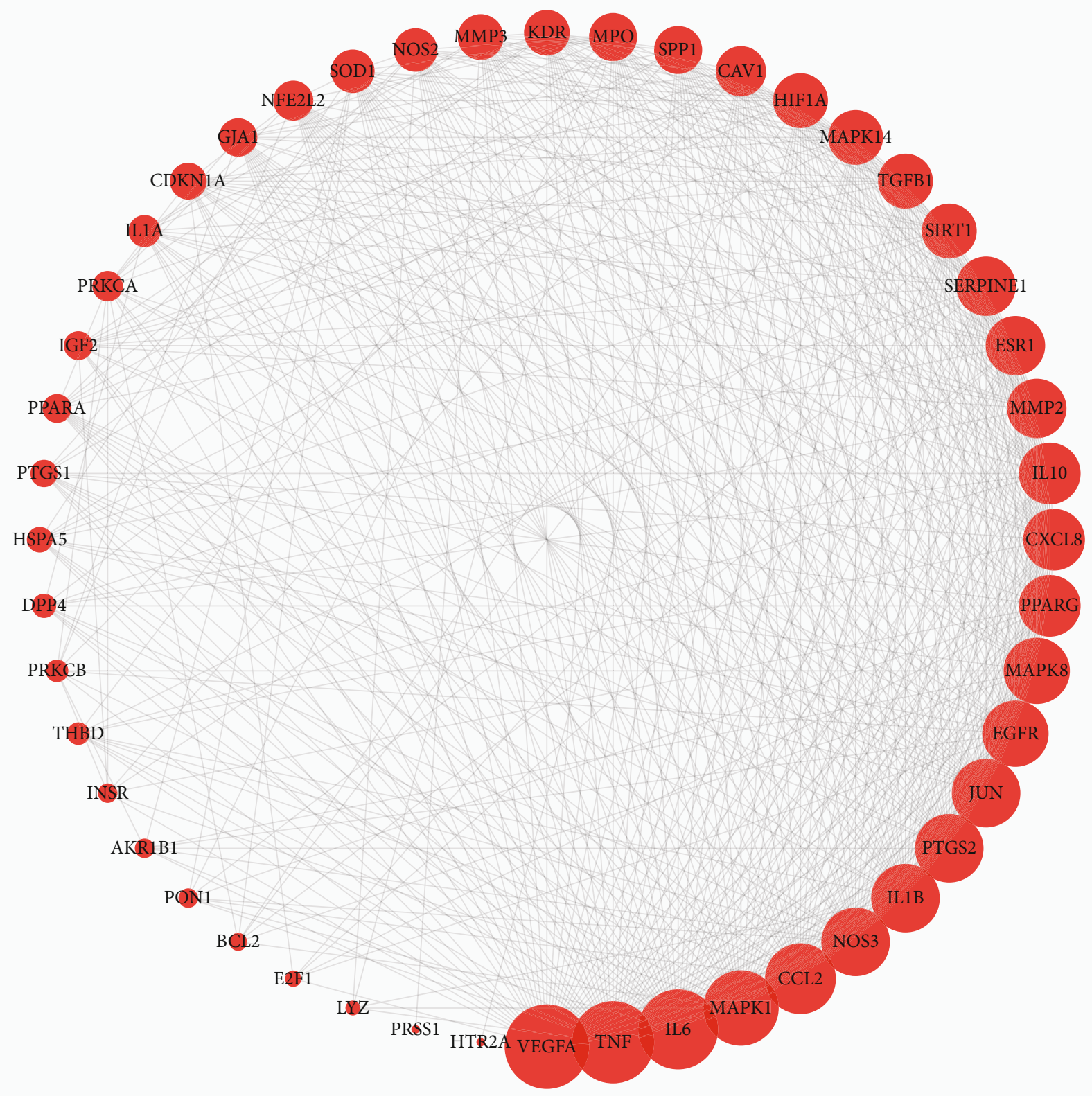

Figure 5: The PPI network of targets for AM in the treatment of DN. The layout of the outer ring is according to the area and color of nodes, which goes better in an anticlockwise direction. The red nodes represent the potential target of AM in DN. The size of the nodes is shown in a gradient from large to small according to the descending order of the degree value. And, the lines among inner nodes display the relationship between different proteins.

results, which indicated that the major hubs were significantly in multiple biological processes, including positive regulation of angiogenesis, lipopolysaccharide-mediated signaling pathway, angiogenesis, positive regulation of endothelial cell proliferation, and positive regulation of peptidylserine phosphorylation, as shown in Figure 4. Furthermore, molecular function enrichment analysis contains identical protein binding, cytokine activity, MAP kinase activity, heme binding, and peroxidase activity, as shown in Figure 5. The active targets involved are TNF, IL-6, MAPK1,
PTGS2, and NOS3, which mainly concentrate on the molecular processes of immune regulation, oxidative stress, inflammatory response, etc. To some extent, this is consistent with the pathogenesis and mechanism of clinical DN. Meanwhile, cellular components consist of extracellular space, membrane raft, cytosol, extracellular matrix, caveola, Golgi apparatus, mitochondrion, and extracellular exosome, and many targets were ranked highly as potential related genes. This indirectly illustrated the complexity of the pathogenesis of DN and the damage to various cellular 


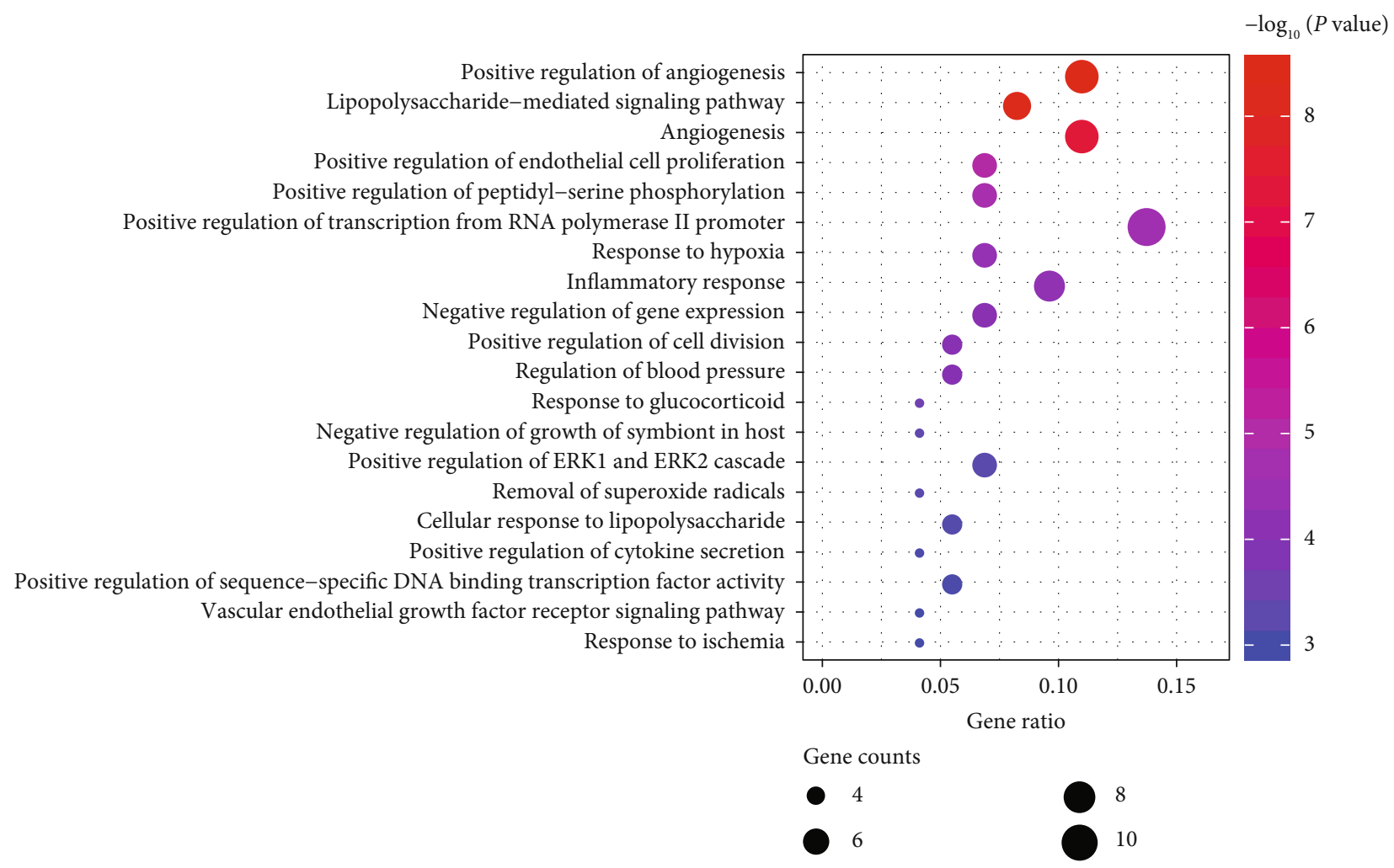

FIGURE 6: Enriched GO terms for biological process (BP) of potential targets of the main active ingredients of AM. The color of the nodes is shown in a gradient from red to blue according to the descending order of the $P$ value. The size of nodes is arranged according to the ascending order of the number of gene counts.

components. For instance, IL-6, CCL2, TNF, DPP4, and HIF- $1 \alpha$ play a key regulatory role in this pathogenic process. Among them, DPP4 target inhibitors have been widely used in the treatment of diabetes mellitus, which has an important impact on reducing blood sugar in patients [32].

HIF-1 signaling pathway activates the expression of VEGFA in inflammatory response to counteract hypoxia and prevent kidney tissue damage in conjunction [33]. VEGFA contributes significantly in the pathogenesis of DN, and overexpression will promote the proliferation of microvascular endothelial cells and aggravate the occurrence of vascular disease. The research suggested that VEGFA could serve as biomarkers to identify the progression of DN and had higher expression in the plasma and urine of DN patients [34]. In addition, after treatment with medicine, not only FBG and Scr levels were decreased but also VEGF, HIF- $1 \alpha$, and TGF- $\beta 1$ mRNA expression was downregulated in DN mice [35]. Meanwhile, NOS3, HIF-1 $\alpha$, and VEGFA play a crucial role in angiogenesis and endothelial cell proliferation. A further investigation showed that VEGF increased hTERT expression in a mechanism that implicates the PI3K/AKT/mTOR pathway and HIF-1 signaling pathway.

In the pathogenesis of DN, inflammatory cells will proliferate and differentiate, increase the infiltration of renal tissue cells, and secrete a large number of cytokines to mediate renal tissue damage. In addition, these inflammatory cells and cytokines secreted by them can stimulate and activate kidney cells, regulating the expression of chemokines, cytokines, adhesion molecules, and extracellular matrix components, and aggravate the pathological changes of kidney tissue. TNF- $\alpha$, one of the main regulatory factors, which can induce the production of IL- $1 \beta$, IL- 6 , and other cytokines, participates in the process of oxidative stress and inflammation and aggravation of renal tissue damage [36]. IL-6, as an autocrine growth factor of mesangial cells, can significantly stimulate mesangial proliferation. The level of IL- 6 in urine can reflect the degree of glomerular and tubulointerstitial lesions, which has guiding significance for the diagnosis of diabetes mellitus [37]. However, treatment of diabetic rats might significantly inhibit the production and release of inflammatory cytokines IL-6, IL- $1 \beta$, and TNF- $\alpha$ into the serum [38]. What is more, in agreement with the improved oxidative response, TNF- $\alpha$, IL- $1 \beta$, and ICAM-1 expression was all markedly blocked in DN mice [39]. Collectively, these inflammatory factors are mainly concentrated in rheumatoid arthritis, FoxO signaling pathway, toll-like receptor signaling pathway, and TNF signaling pathway, and aggravated the symptoms of DN to some extent.

MAPK1 is an important transmitter of signals from the cell surface to the nucleus in NOD-like receptor signaling pathway. It can activate the release of various inflammatory factors, chemokines, and adhesion factors mediated by NF$\mathrm{KB}$ signaling pathways and aggravate the pathological changes of DN [40]. When inflammatory reaction happens, MAPK1 will promote IL- 1 , TNF- $\alpha$, and IL- $1 \beta$ entry into glomerular cells by activating nod-like receptor signaling pathway and increase cell damage. CCL2 is a secretory protein that mediates the phosphorylation of C-terminal 

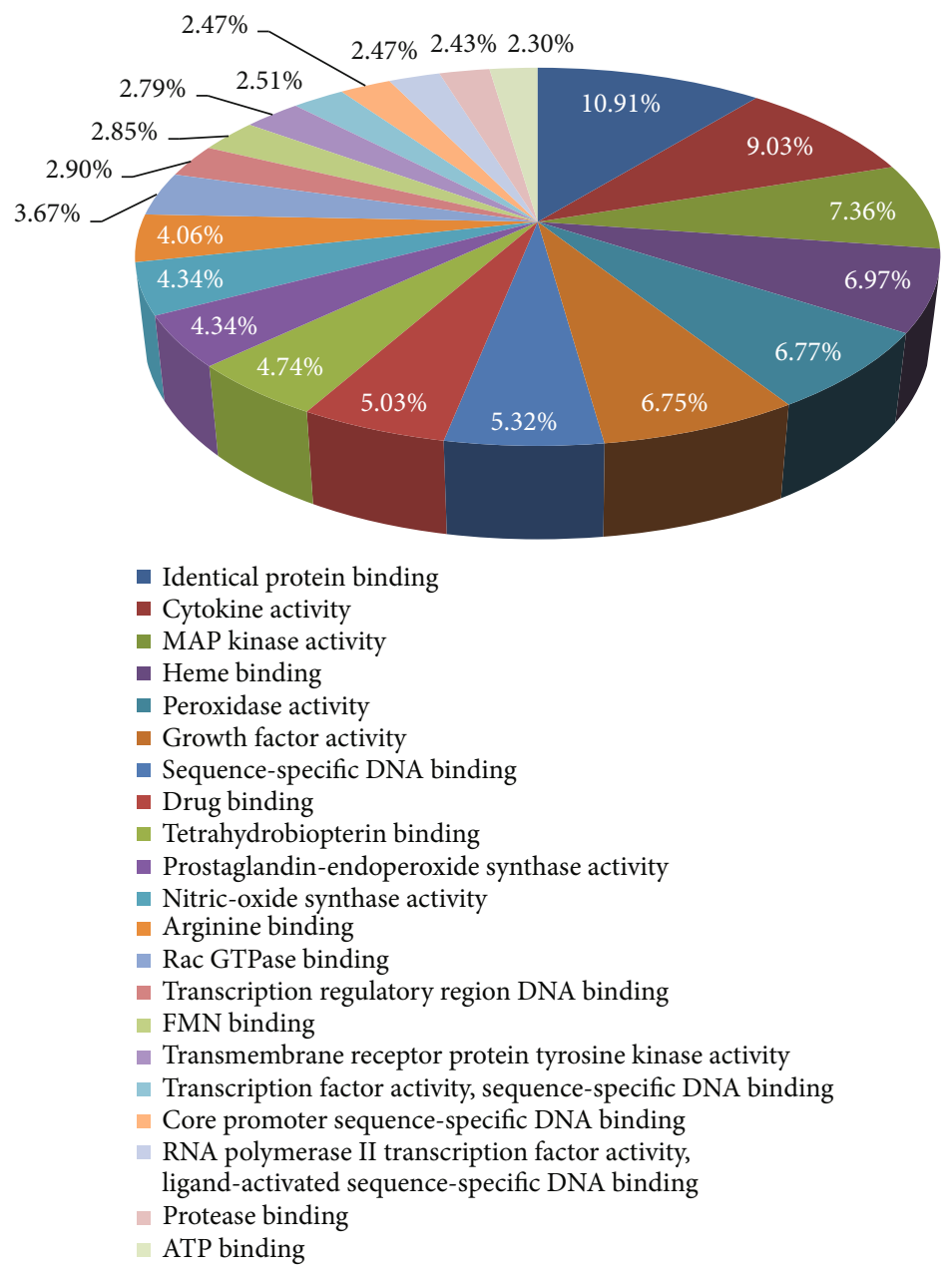

FIGURE 7: Enriched GO terms for molecular function (MF) of potential targets of the main active ingredients of AM. The percentage of MF is clockwise arranged according to the descending order of the $P$ value.

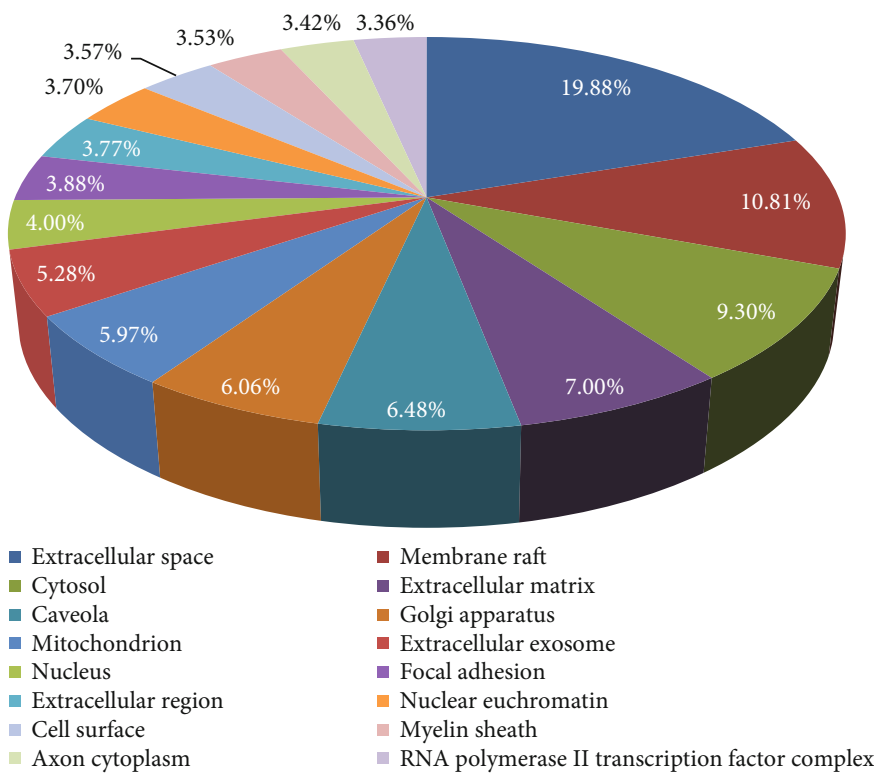

FIGURE 8: Enriched GO terms for cell component (CC) of potential targets of the main active ingredients of AM. The percentage of CC is clockwise arranged according to the descending order of the $P$ value. 


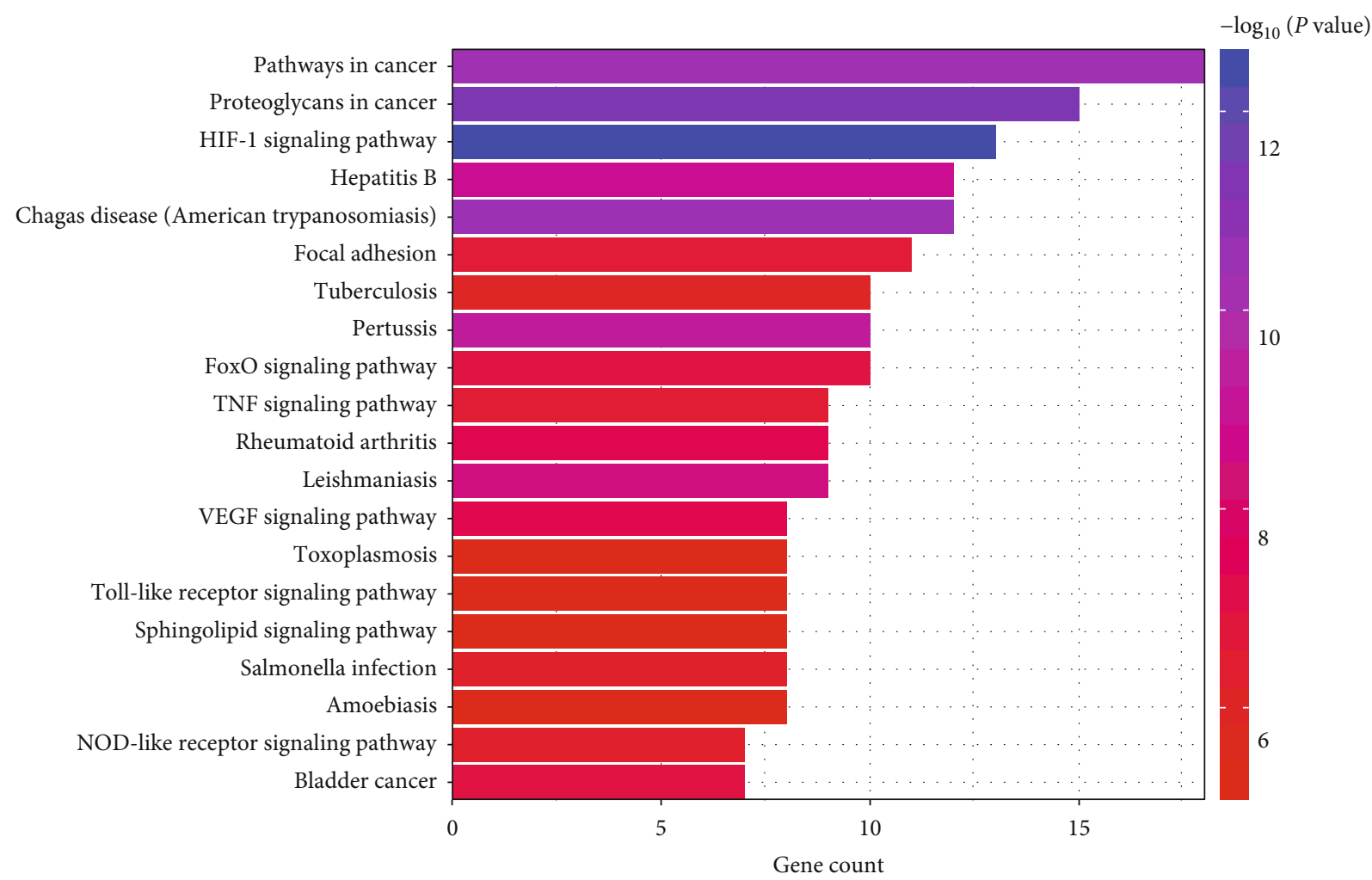

FIgURE 9: KEGG pathway analysis of putative target genes of AM. The color of the item is shown in a gradient from blue to red according to the descending order of the $P$ value. The length of item is arranged according to the ascending order of the number of gene counts.

serine/threonine of receptor protein by binding to the $\mathrm{N}$ terminal of CCR2 on target cell membrane [41]. It participates in various physiological and pathological activities of cells through different transmembrane signal transduction pathways. It can migrate chemokine cells and make immune cells migrate along the site where chemokine concentration increases. The release of chemokines can also stimulate the expression of inflammatory cytokines, such as IL-1, IL-6, and TNF- $\alpha$, which can repair infection or tissue damage [42]. Therefore, these results also confirm that our screened targets are consistent with the literature reports, indicating that $\mathrm{AM}$ can play a therapeutic role in $\mathrm{DN}$ by regulating inflammatory response, oxidative stress, and other pathways.

\section{Conclusion}

In summary, AM has significant advantages in the treatment of $\mathrm{DN}$, which is consistent with previous studies. At the same time, the biological functions of active ingredients and their corresponding targets of AM were analyzed by network pharmacological method, which further revealed the molecular biological mechanism of AM in treating DN. It has a significant value to provide theoretical basis for clinical treatment of DN.

\section{Data Availability}

The data used to support the findings of this study are available from the corresponding author upon request.

\section{Conflicts of Interest}

The authors declare that they have no conflicts of interest.

\section{Authors' Contributions}

MF $G$ conceived and designed the study. MF $G$ and $Y J$ $\mathrm{D}$ wrote the paper. JR G and PJ $\mathrm{C}$ reviewed and edited the manuscript. All authors read and approved the manuscript.

\section{Acknowledgments}

This research was supported by the National Natural Science Foundation of China (Grant No. 81973546) and the second batch of Scientific research projects of the national TCM clinical research base of the State Administration of traditional Chinese medicine (Grant No. JDZX2015126). We are grateful to Mr. Yan Li (The Fourth Affiliated Hospital of Anhui Medical University, Hefei, China) for providing help in data analysis.

\section{Supplementary Materials}

Supplementary 1. Table S1: Compound targets for each component in AM.

Supplementary 2. Table S2: Screening of common targets for DN and AM. 


\section{References}

[1] Y. Song, W. Liu, K. Tang, J. Zang, D. Li, and H. Gao, "Mangiferin alleviates renal interstitial fibrosis in streptozotocininduced diabetic mice through regulating the PTEN/PI3K/Akt signaling pathway," Journal of Diabetes Research, vol. 2020, Article ID 9481720, 12 pages, 2020.

[2] S. Jiang, T. Yu, Z. Zhang et al., "Prognostic nomogram and score to predict renal survival of patients with biopsy-proven diabetic nephropathy," Diabetes Research and Clinical Practice, vol. 155, article 107809, 2019.

[3] A. Abdel-Moneim, B. Mahmoud, A. Nabil, and Z. Negeem, "Correlation between oxidative stress and hematological profile abnormalities in diabetic nephropathy," Diabetes and Metabolic Syndrome: Clinical Research and Reviews, vol. 13, no. 4, pp. 2365-2373, 2019.

[4] G. Wang, J. Ouyang, S. Li et al., "The analysis of risk factors for diabetic nephropathy progression and the construction of a prognostic database for chronic kidney diseases," Journal of Translational Medicine, vol. 17, no. 1, article 264, 2019.

[5] G. Chen, X. Yang, X. Yang et al., "Jia-Wei-Jiao-Tai-Wan ameliorates type 2 diabetes by improving $\beta$ cell function and reducing insulin resistance in diabetic rats," BMC Complementary and Alternative Medicine, vol. 17, no. 1, article 507, 2017.

[6] T. Nozaki, J. Minaguchi, K. Takehana, and H. Ueda, “Anti-diabetic activities of traditional Chinese herbal medicine in streptozotocin-induced diabetic rats," Okajimas Folia Anatomica Japonica, vol. 93, no. 4, pp. 111-118, 2017.

[7] H. Liao, L. Hu, X. Cheng et al., "Are the therapeutic effects of Huangqi (Astragalus membranaceus) on diabetic nephropathy correlated with its regulation of macrophage iNOS activity?," Journal of Immunology Research, vol. 2017, Article ID 3780572, 9 pages, 2017.

[8] K. Q. He, W. Z. Li, X. Q. Chai, Y. Y. Yin, Y. Jiang, and W. P. Li, "Astragaloside IV prevents kidney injury caused by iatrogenic hyperinsulinemia in a streptozotocin-induced diabetic rat model," International Journal of Molecular Medicine, vol. 41, no. 2, pp. 1078-1088, 2018.

[9] S. Adesso, R. Russo, A. Quaroni, G. Autore, and S. Marzocco, "Astragalus membranaceus extract attenuates inflammation and oxidative stress in intestinal epithelial cells via NF- $\kappa \mathrm{B}$ activation and Nrf2 response," International Journal of Molecular Sciences, vol. 19, no. 3, article 800, 2018.

[10] M. R. Farag, W. M. Elhady, S. Y. A. Ahmed, H. S. A. Taha, and M. Alagawany, "Astragalus polysaccharides alleviate tilmicosin-induced toxicity in rats by inhibiting oxidative damage and modulating the expressions of HSP70, NF-kB and Nrf2/HO-1 pathway," Research in Veterinary Science, vol. 124, pp. 137-148, 2019.

[11] J. Zhang, R. Liang, L. Wang, and B. Yang, "Effects and mechanisms of Danshen-Shanzha herb-pair for atherosclerosis treatment using network pharmacology and experimental pharmacology," Journal of Ethnopharmacology, vol. 229, pp. 104-114, 2019.

[12] L. Chen, Y. Cao, H. Zhang et al., "Network pharmacologybased strategy for predicting active ingredients and potential targets of Yangxinshi tablet for treating heart failure," Journal of Ethnopharmacology, vol. 219, pp. 359-368, 2018.

[13] W. Song, S. Ni, Y. Fu, and Y. Wang, "Uncovering the mechanism of Maxing Ganshi Decoction on asthma from a systematic perspective: A network pharmacology study," Scientific Reports, vol. 8, no. 1, article 17362, 2018.
[14] J. Ru, P. Li, J. Wang et al., "TCMSP: a database of systems pharmacology for drug discovery from herbal medicines," Journal of Cheminformatics, vol. 6, no. 1, article 13, 2014.

[15] B. Zhu, W. Zhang, Y. Lu et al., "Network pharmacology-based identification of protective mechanism of Panax notoginseng saponins on aspirin induced gastrointestinal injury," Biomedicine \& Pharmacotherapy, vol. 105, pp. 159-166, 2018.

[16] S.-J. Yue, J. Liu, W.-W. Feng et al., "System pharmacologybased dissection of the synergistic mechanism of Huangqi and Huanglian for diabetes mellitus," Frontiers in Pharmacology, vol. 8, article 694, 2017.

[17] X. X. Xu, J. P. Bi, L. Ping, P. Li, and F. Li, “A network pharmacology approach to determine the synergetic mechanisms of herb couple for treating rheumatic arthritis," Drug Design, Development and Therapy, vol. 12, pp. 967-979, 2018.

[18] T. Yu, A. Acharya, N. Mattheos et al., "Molecular mechanisms linking peri-implantitis and type 2 diabetes mellitus revealed by transcriptomic analysis," PeerJ, vol. 7, article e7124, 2019.

[19] S. Paolacci, V. Precone, F. Acquaviva et al., "Genetics of lipedema: new perspectives on genetic research and molecular diagnoses," European Review for Medical and Pharmacological Sciences, vol. 23, no. 13, pp. 5581-5594, 2019.

[20] L. Y. Zeng, Q. Wang, L. Y. Han et al., "Mechanism of tripterygium hypoglaucum-leonuri Herba for treating rheumatoid arthritis based on network pharmacology," Chinese Journal of Experimental Traditional Medical Formulae, vol. 25, no. 19, pp. 170-181, 2019.

[21] C. Huang, R. Li, W. Shi, and Z. Huang, "Discovery of the antitumor mechanism of calycosin against colorectal cancer by using system pharmacology approach," Medical Science Monitor, vol. 25, pp. 5589-5593, 2019.

[22] T. Qin, L. Wu, Q. Hua, Z. Song, Y. Pan, and T. Liu, "Prediction of the mechanisms of action of Shenkang in chronic kidney disease: a network pharmacology study and experimental validation," Journal of Ethnopharmacology, vol. 246, article 112128, 2020.

[23] W. Wang, T. Wang, Y. Wang et al., "Integration of gene expression profile data to verify hub genes of patients with Stanford a aortic dissection," BioMed Research International, vol. 2019, Article ID 3629751, 9 pages, 2019.

[24] J. L. Deng, Y. H. Xu, and G. Wang, "Identification of potential crucial genes and key pathways in breast cancer using bioinformatic analysis," Frontiers in Genetics, vol. 10, p. 695, 2019.

[25] H. Dai, Q. Liu, and B. Liu, "Research progress on mechanism of podocyte depletion in diabetic nephropathy," Journal of Diabetes Research, vol. 2017, Article ID 2615286, 10 pages, 2017.

[26] C. Zhang, X. Zhu, L. Li et al., "A small molecule inhibitor MCC950 ameliorates kidney injury in diabetic nephropathy by inhibiting NLRP3 inflammasome activation," Diabetes, Metabolic Syndrome and Obesity: Targets and Therapy, vol. 12, pp. 1297-1309, 2019.

[27] S. Di, L. Han, Q. Wang et al., "A network pharmacology approach to uncover the mechanisms of Shen-Qi-Di-Huang decoction against diabetic nephropathy," Evidence-Based Complementary and Alternative Medicine, vol. 2018, Article ID 7043402, 14 pages, 2018.

[28] D. Lei, L. Chengcheng, Q. Xuan et al., "Quercetin inhibited mesangial cell proliferation of early diabetic nephropathy through the Hippo pathway," Pharmacological Research, vol. 146, article 104320, 2019. 
[29] H. Iskender, E. Dokumacioglu, T. M. Sen, I. Ince, Y. Kanbay, and S. Saral, "The effect of hesperidin and quercetin on oxidative stress, NF- $\kappa$ B and SIRT1 levels in a STZ-induced experimental diabetes model," Biomedicine \& Pharmacotherapy, vol. 90, pp. 500-508, 2017.

[30] Y. Zhang, C. Chen, and J. Zhang, "Effects and significance of formononetin on expression levels of HIF- $1 \alpha$ and VEGF in mouse cervical cancer tissue," Oncology Letters, vol. 18, no. 3, pp. 2248-2253, 2019.

[31] S. K. L. Ong, M. K. Shanmugam, L. Fan et al., "Focus on formononetin: anticancer potential and molecular targets," Cancers, vol. 11, no. 5, article 611, 2019.

[32] S. Gupta and U. Sen, "More than just an enzyme: dipeptidyl peptidase-4 (DPP-4) and its association with diabetic kidney remodelling," Pharmacological Research, vol. 147, article 104391, 2019.

[33] R. Bohuslavova, R. Cerychova, K. Nepomucka, and G. Pavlinkova, "Renal injury is accelerated by global hypoxia-inducible factor 1 alpha deficiency in a mouse model of STZ-induced diabetes," BMC Endocrine Disorders, vol. 17, no. 1, p. 48, 2017.

[34] D. Kallinikou, A. Soldatou, C. Tsentidis et al., "Diabetic neuropathy in children and adolescents with type 1 diabetes mellitus: diagnosis, pathogenesis, and associated genetic markers," Diabetes/Metabolism Research and Reviews, vol. 35, no. 7, article e3178, 2019.

[35] X. Lai, D. Tong, X. Ai et al., “Amelioration of diabetic nephropathy in $\mathrm{db} / \mathrm{db}$ mice treated with Tibetan medicine formula Siwei Jianghuang Decoction Powder extract," Scientific Reports, vol. 8, no. 1, article 16707, 2018.

[36] Q. Ge, L. Chen, M. Tang et al., "Analysis of mulberry leaf components in the treatment of diabetes using network pharmacology," European Journal of Pharmacology, vol. 833, pp. 5062, 2018.

[37] D. D. Chen, R. Xu, J. Y. Zhou et al., "Cordyceps militaris polysaccharides exerted protective effects on diabetic nephropathy in mice via regulation of autophagy," Food \& Function, vol. 10, no. 8, pp. 5102-5114, 2019.

[38] F. Ma, L. Li, Q. Wang et al., "Qi-dan-di-huang decoction alleviates diabetic nephropathy by inhibiting the NF-kappaB pathway," Frontiers in Bioscience, vol. 24, no. 8, pp. 14771486, 2019.

[39] M. Wu, S. Li, X. Yu et al., "Mitochondrial activity contributes to impaired renal metabolic homeostasis and renal pathology in STZ-induced diabetic mice," American Journal of Physiology. Renal Physiology, vol. 317, no. 3, pp. F593-F605, 2019.

[40] C. Jiang, M. Xu, X. Kuang et al., “Treponema pallidum flagellins stimulate MMP-9 and MMP-13 expression via TLR5 and MAPK/NF- $\kappa$ B signaling pathways in human epidermal keratinocytes," Experimental Cell Research, vol. 361, no. 1, pp. 46$55,2017$.

[41] J. A. Moreno, C. Gomez-Guerrero, S. Mas et al., "Targeting inflammation in diabetic nephropathy: a tale of hope," Expert Opinion on Investigational Drugs, vol. 27, no. 11, pp. 917930, 2018.

[42] M. Tziastoudi, I. Stefanidis, G. M. Hadjigeorgiou, K. Stravodimos, and E. Zintzaras, "A systematic review and meta-analysis of genetic association studies for the role of inflammation and the immune system in diabetic nephropathy," Clinical Kidney Journal, vol. 10, no. 3, pp. 293-300, 2017. 